\title{
Assessing different imaging velocimetry techniques to measure shallow runoff velocities during rain events using an urban drainage physical model
}

\author{
Juan Naves ${ }^{1}$, Juan T. García ${ }^{2}$, Jerónimo Puertas ${ }^{1}$, Joaquín Suárez ${ }^{1}$, Jose Anta ${ }^{1}$ \\ ${ }^{1}$ Universidade da Coruña, Water and Environmental Engineering Research Team (GEAMA), Civil \\ Engineering School, A Coruña, 15071, Spain \\ ${ }^{2}$ Mining and Civil Engineering Department, Universidad Politécnica de Cartagena, Cartagena, 30203, \\ Spain \\ Correspondence to: Juan Naves (juan.naves@udc.es)
}




\section{S1. Complementary results for the sensitivity analysis}

$30 \mathrm{~mm} \mathrm{~h}^{-1}$-rain-intensity experiments

- - Pre-processing parameter $\left(X_{\text {ref_LSPIV }}=25 \%, X_{\text {ref_LSPIV+ }}=0.25, X_{\text {ref_SSIV }}=15 \%, X_{\text {ref_BIV }}=0.6\right.$ )

- - IA size $\left(X_{\text {ref }}=32 \mathrm{px}\right)$

$\rightarrow$ FAR $\left(X_{\text {ref }}=25 \mathrm{~Hz}\right)$
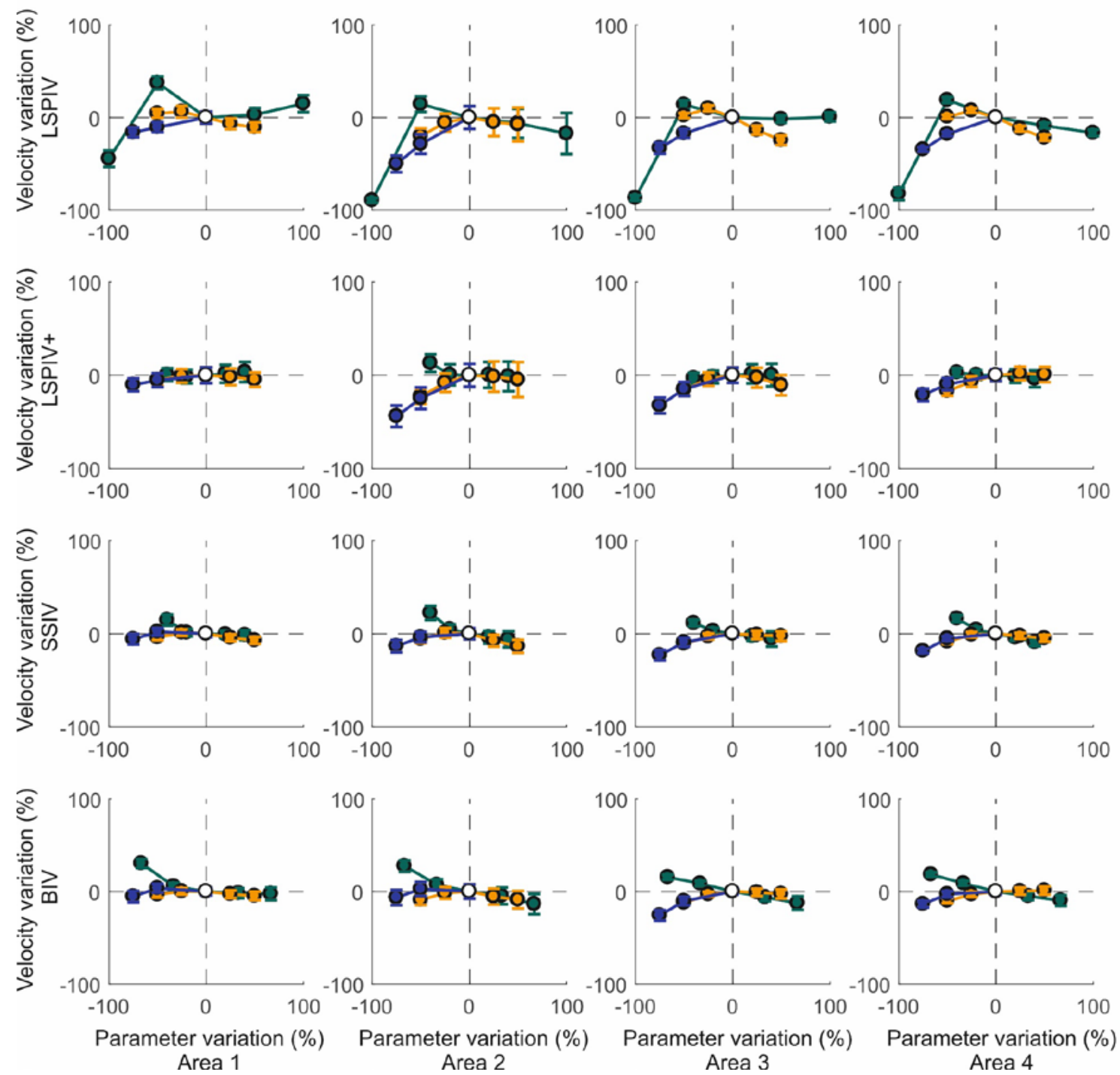

Figure S1: Percentage of variation in the mean velocities when varying parameters of the analysis for the four studied areas (columns) and the four imaging velocimetry techniques considered (rows) in the case of $30 \mathrm{~mm} \mathrm{~h}^{-}$ ${ }^{1}$ rainfall. Mean velocity variability for the different pairs of frames analyzed are included using whiskers. 


\section{$80 \mathrm{~mm} \mathrm{~h}^{-1}$-rain-intensity experiments}

- Pre-processing parameter $\left(X_{\text {ref_LSPIV }}=25 \%, X_{\text {ref_LSPIV+ }}=0.25, X_{\text {ref_SSIV }}=15 \%, X_{\text {ref_BIV }}=0.6\right.$ )

- - IA size $\left(X_{\text {ref }}=32 \mathrm{px}\right)$

$\rightarrow \operatorname{FAR}\left(\mathrm{X}_{\text {ref }}=25 \mathrm{~Hz}\right)$
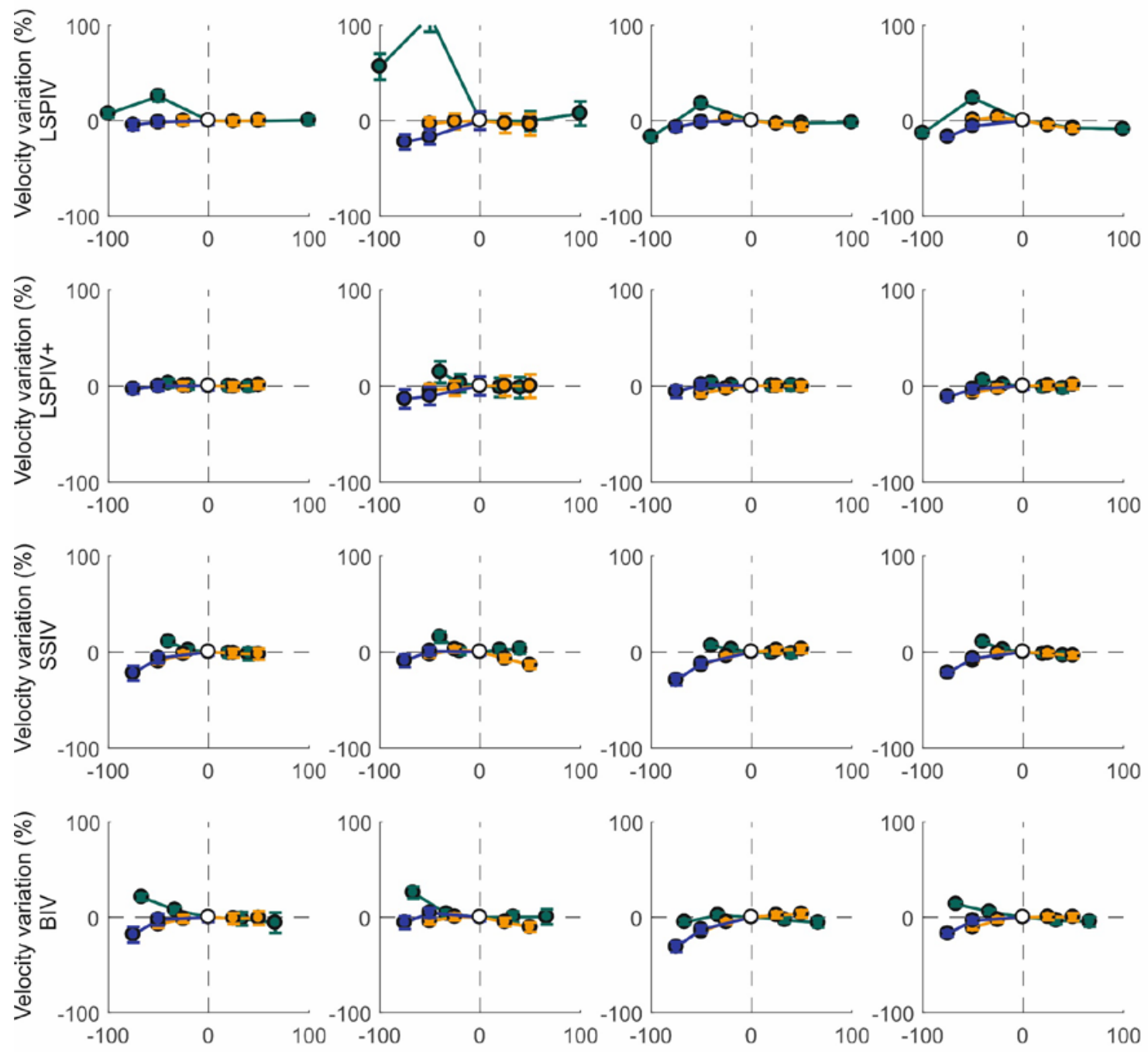

Parameter variation (\%) Parameter variation (\%)

Parameter variation $(\%)$

Parameter variation (\%) Area 1 Area 2 Area 3

Area 4

Figure S2: Percentage of variation in the mean velocities when varying parameters of the analysis for the four studied areas (columns) and the four imaging velocimetry techniques considered (rows) in the case of $80 \mathbf{~ m m ~ h}^{-}$ ${ }^{1}$ rainfall. Mean velocity variability for the different pairs of frames analyzed are included using whiskers. 


\section{S2. Complementary results for the velocity results comparison}

Velocity comparison Area 3

$\underline{30 \mathrm{~mm} \mathrm{~h}^{-1}}$

LSPIV

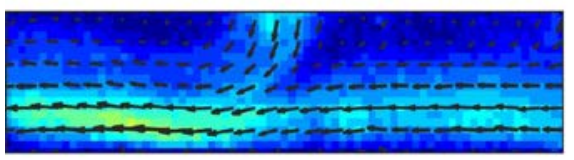

LSPIVb

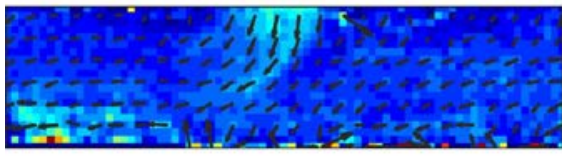

SSIV

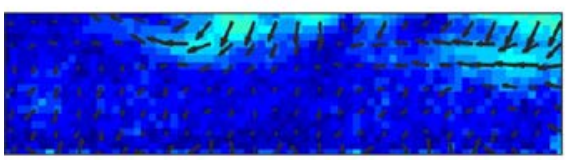

BIV

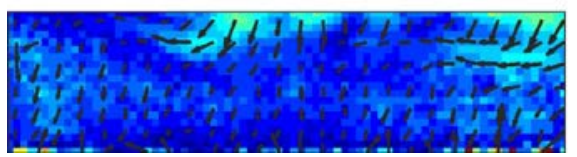

Velocity $(\mathrm{m} / \mathrm{s})$ :

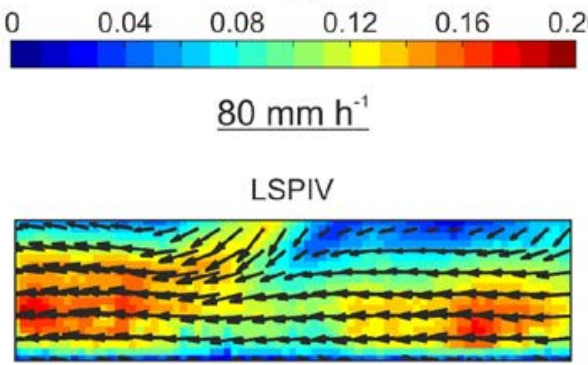

LSPIVb

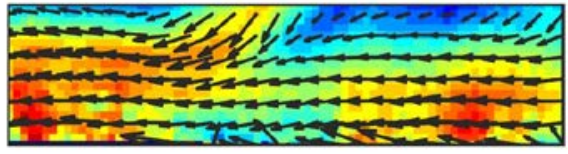

SSIV

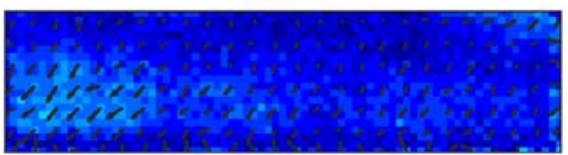

BIV

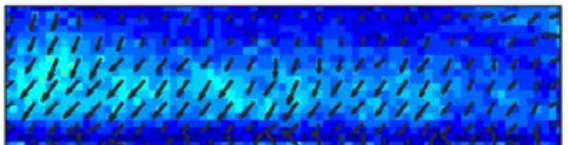

Figure S3: Velocity fields obtained for the cases of $30 \mathrm{~mm} \mathrm{~h}^{-1}$ and $80 \mathrm{~mm} \mathrm{~h}^{-1}$ in Area 3.

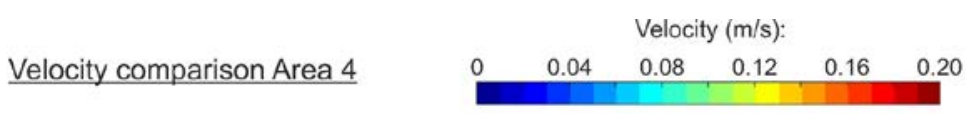

$30 \mathrm{~mm} \mathrm{~h}^{-1}$

LSPIV

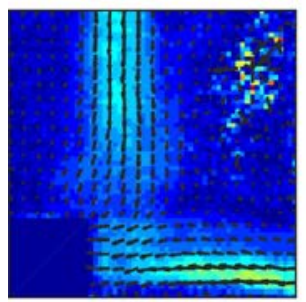

SSIV

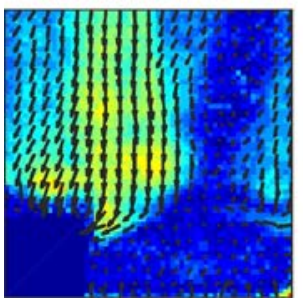

LSPIVb

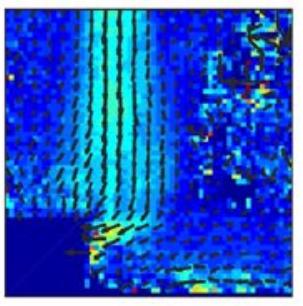

BIV

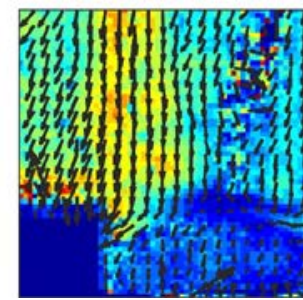

$80 \mathrm{~mm} \mathrm{~h}^{\circ}$

LSPIV

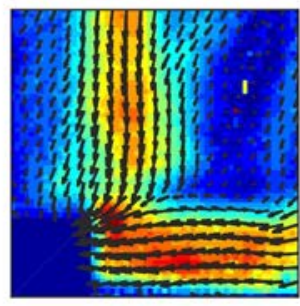

SSIV

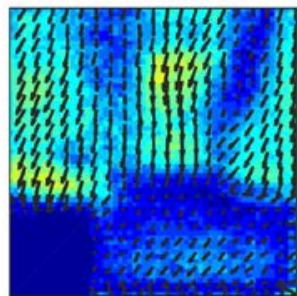

LSPIVb

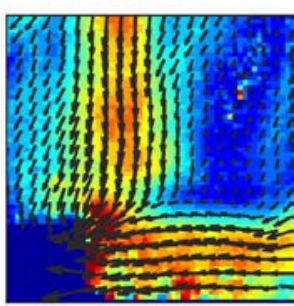

BIV

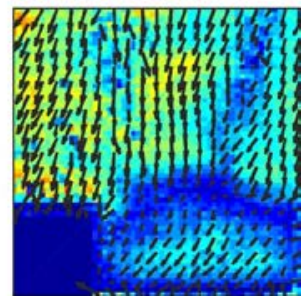

Figure S4: Velocity fields obtained for the cases of $30 \mathrm{~mm} \mathrm{~h}^{-1}$ and $80 \mathrm{~mm} \mathrm{~h}^{-1}$ in Area 4. 


\section{S3. Complementary results for the convergence study}

Convergence study: mean velocities (rain intensity $=30 \mathrm{~mm} \mathrm{~h}^{-1}$ )

- LSPIV $\quad \bullet \quad$ LSPIVb
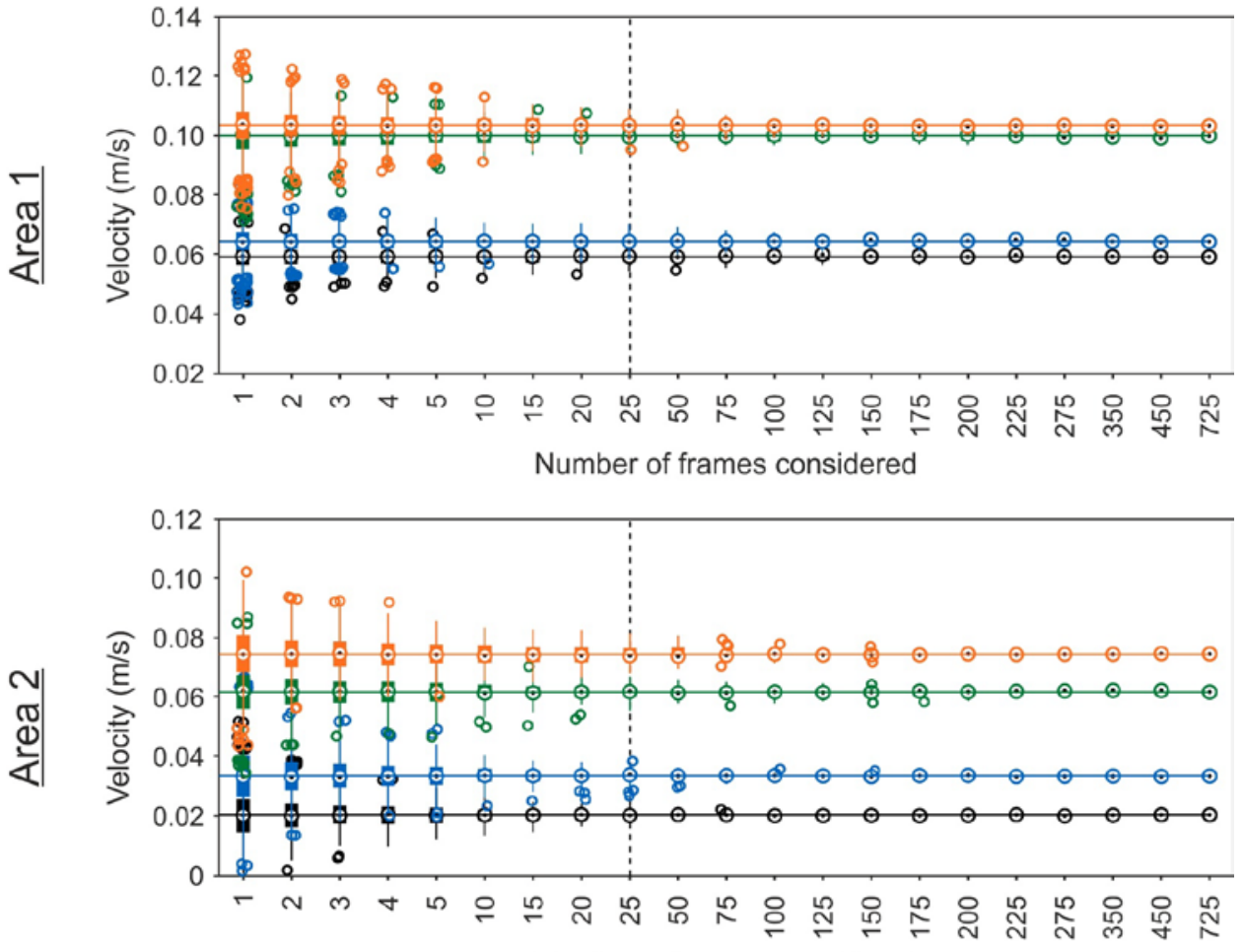

Number of frames considered

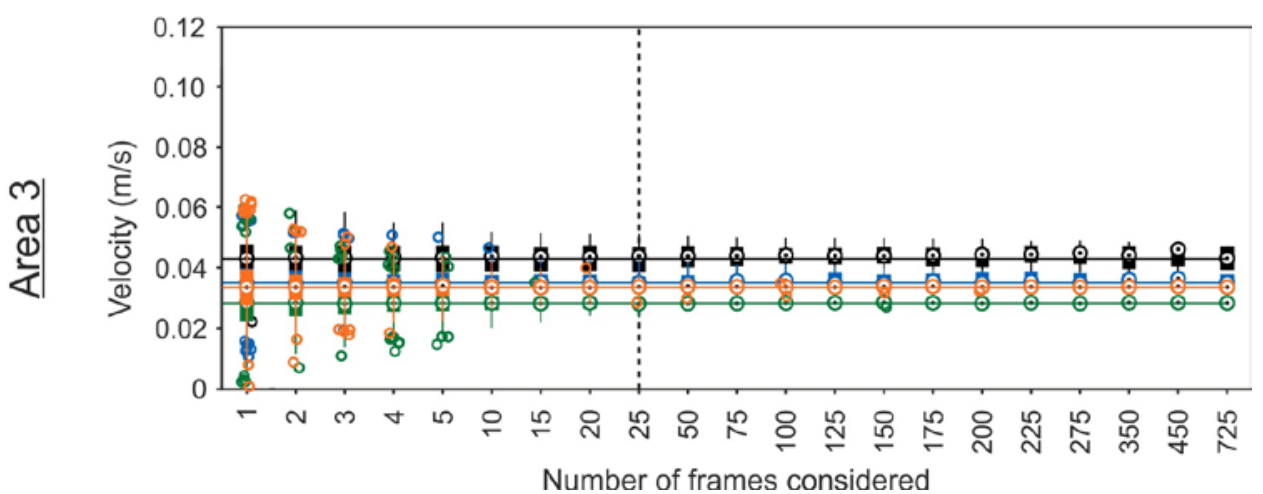

Figure S5: Mean velocity convergence study for rain intensity of $30 \mathrm{~mm} \mathrm{~h}^{-1}$ and Areas 1, 2, and 3. The horizontal line represents the mean velocity considering all the frames available (1500) for LSPIV (black), LSPIVb (blue), SSIV (green) and BIV (orange) techniques. Then, the variability in the mean velocity when the frames are divided into groups of different numbers of frames was represented by boxplots. 
Convergence study: mean velocities (rain intensity $=80 \mathrm{~mm} \mathrm{~h}^{-1}$ )

- SSIV

BIV
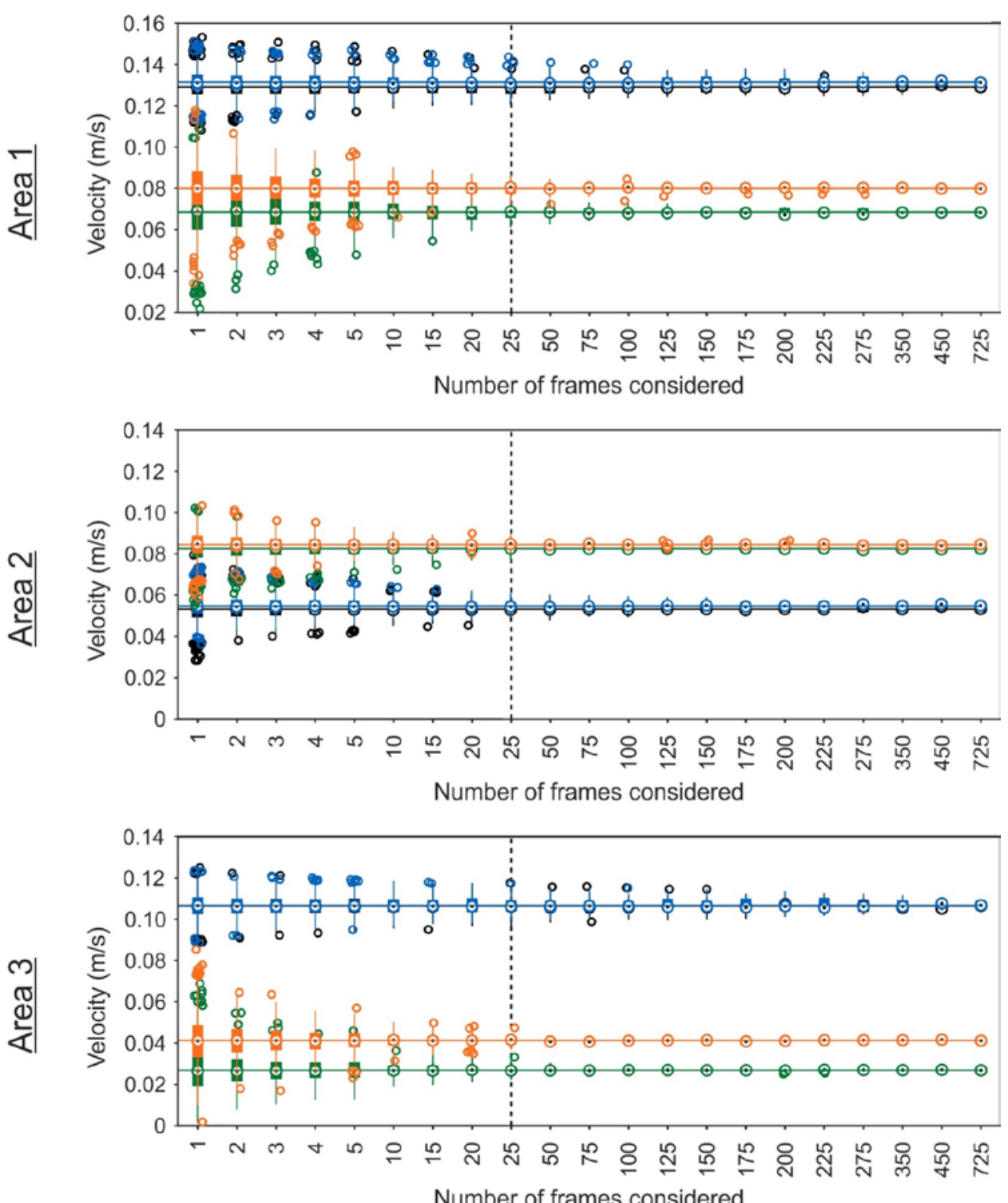

Figure S6: Mean velocity convergence study for rain intensity of $80 \mathrm{~mm} \mathrm{~h}^{-1}$ and Areas 1, 2, and 3. The horizontal line represents the mean velocity considering all the frames available (1500) for LSPIV (black), LSPIVb (blue), SSIV (green) and BIV (orange) techniques. Then, the variability in the mean velocity when the frames are divided into groups of different numbers of frames was represented by boxplots. 
$\bullet \quad$ LSPIV LSPIVb $\bullet$ SSIV

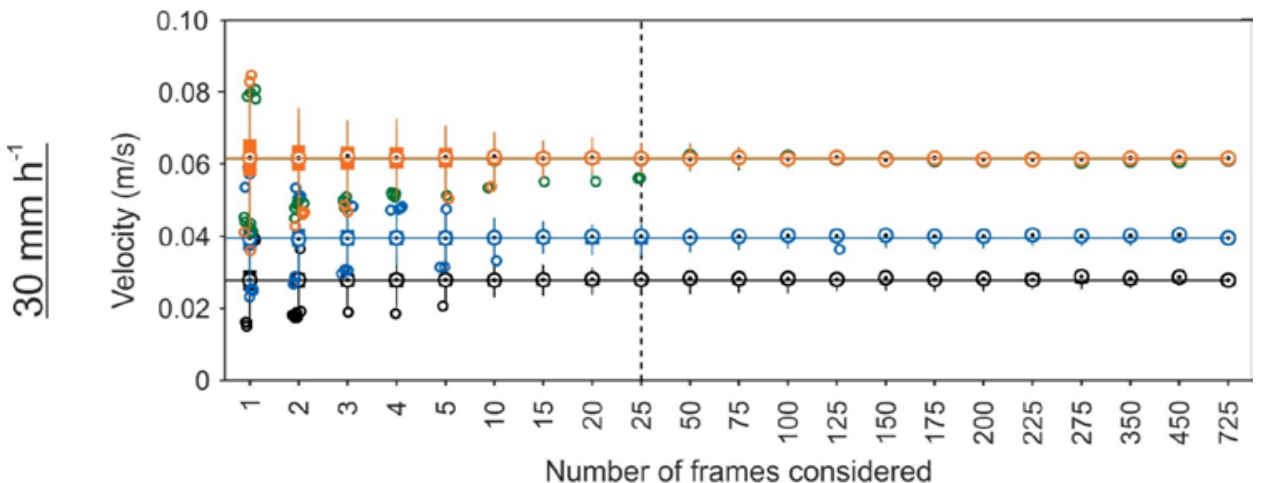

Number of frames considered

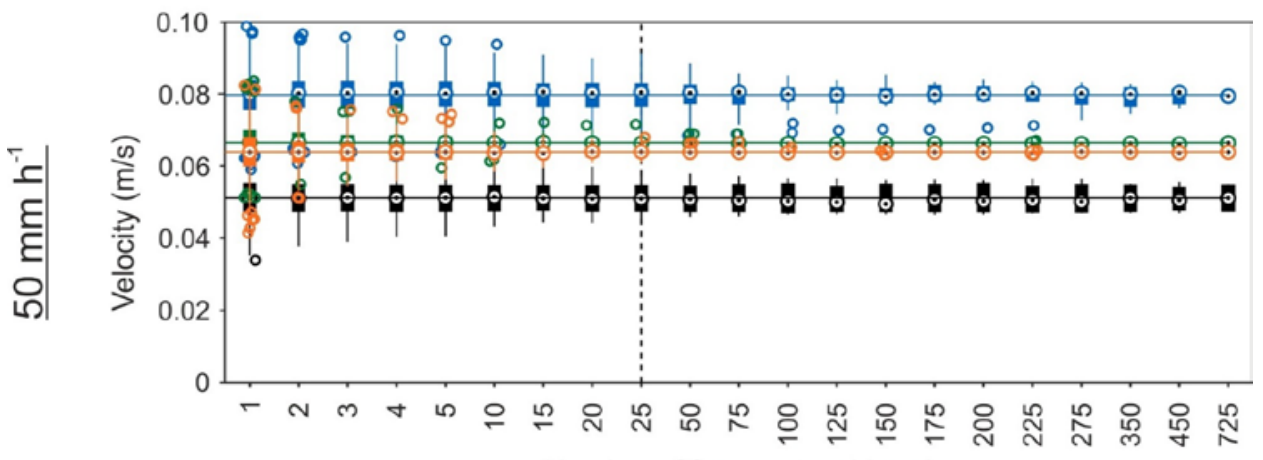

Number of frames considered

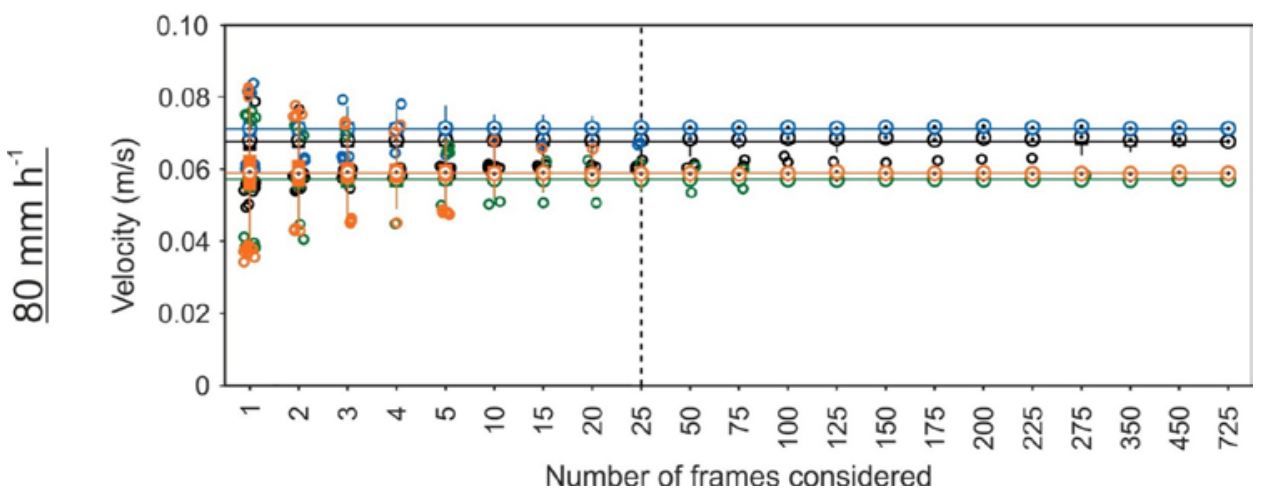

Figure S7. Mean velocity convergence study for Area 4 and rain intensities of 30, 50 and $80 \mathrm{~mm} \mathrm{~h}^{-1}$. The horizontal line represents the mean velocity considering all the frames available (1500) for LSPIV (black), LSPIVb (blue), SSIV (green) and BIV (orange) techniques. Then, the variability in the mean velocity when the frames are divided into groups of different numbers of frames was represented by boxplots. 